\title{
Deep hypometabolic state mitigates radiation-induced lethality in zebrafish
}

\author{
Subhajit Ghosh ${ }^{1,2}$, Namita Indracanti' ${ }^{1}$ Jharna Ray $^{2}$ and Indracanti PremKumar ${ }^{*}$ \\ ${ }^{1}$ Drug Repurposing \&Translational Research Lab, Institute of Nuclear Medicine and Allied Sciences, India \\ ${ }^{2}$ S. N. Pradhan Centre for Neuroscience, University of Calcutta, Kolkata, India
}

\begin{abstract}
The increased risks of ionizing radiation exposure both due to accidental and malevolent terrorist acts demand efficient prophylactics and mitigators. As of now there are no clinically safe and effective radiomitigators. Deep hypometabolic (DH) or suspended animation (SA)-like state render organism's resistant to a variety of stresses. The present study aims at investigating the radiomitigative effect of anoxia-mediated DH state using developing zebrafish as a vertebrate model. Anoxia efficiently induced a DH state (heartbeat, movement and developmental arrest) in developing zebrafish and effects reversed when the embryos brought back to a normoxic environment. Embryos of $4 \mathrm{hpf}$ (hours post-fertilization) in the hypometabolic state effectively tolerated the effects of supra-lethal doses (20 Gy; LD80/6) gamma radiation and rescued from radiation-induced pericardial edema, microopthlamia, microcephaly, skin roughness, bradycardia and lethality (80\% survival advantage after 6 days, over zero survival in radiation treatment alone). The DH-like state induced immediately after exposure to 20 Gy gamma radiation delayed as well as minimized the damage manifestations, and the extent of the delay was found to be equal to the duration for which the embryos were in DH state. The present study demonstrates that the $\mathrm{DH}$ state protects zebrafish from radiation-induced lethality and also mitigates radiation-induced damaging effects when induced prior to or after lethal radiation exposures, respectively.
\end{abstract}

\section{Introduction}

Free radical-mediated damage to macromolecules, altered cell signaling, perturbed cellular and tissue homeostasis leading to cell death are predominant mechanisms through which low linear energy transfer (LET) radiation inflicts damage in biological systems [1-3]. The extent of damage manifestation and its consequences during the post-irradiation (post-IR) period depends upon the absorbed dose and elapsed time, and damage manifestation becomes irreversible with time [4]. It can be expected that if the rate of cellular metabolism is reduced, it would lead to slowing down of molecular and biochemical activities and ultimately damage manifestation in irradiated organisms. Reduction in the rate of metabolism, by lowering temperature, is a well-studied and is known to reduce radiation-mediated damage [5]. Oxygen, available in the cell and tissue milieu, further complex's radiation damage by increasing the quality and quantity of free radicals and the decreasing oxygen availability is known to reduce the flux of oxygen centered free radicals and subsequent damage induction [6]. In nature, a number of vertebrates and invertebrates undergo metabolically suppressed state known by a variety of terms like hibernation, torpor, aestivation and brumation [7]. During this state oxygen requirement as well as the metabolic rate decreases to a minimum level where the organism operates cellular function, which is just sufficient for survival $[8,9]$. Experimental studies have clearly suggested that naturally nonhibernating species like laboratory mice can also be made to enter the hibernation-like state by a variety of approaches like low temperature, hypoxia, hydrogen sulfide, carbon monoxide, etc. [10]. One of the hallmarks of the hypometabolic state is to render the organism resistant to a variety of stresses [11-15]. These studies have raised significant interest in the phenomenon of hypometabolism and its possible biomedical implications [16]. The controlled and reversible slowing down of the metabolism and subsequent molecular functions has interesting implications for radiation exposure scenarios [16]. Young and Taylor [17] have suggested that in humans, a wakeful hypometabolic state is possible through parasympathetic dominance during meditation, while the cardiac activity and other biochemical data supported biological aestivation. It can be anticipated that a reversible hypometabolic state, if induced in radiation exposure victims, will reduce the radiation effects which would be invaluable for management of radiation exposed victims. In the recent past, zebrafish, a tropical minnow, have been widely used as a model for understanding basic pathophysiology and also for screening of drugs for a variety of diseases [18]. Some of the important features which make zebrafish an attractive model includes rapid development, transparent early developmental stages, ease of maintenance and high fecundity [19]. The present study was undertaken to investigate whether a reversible hypometabolic state renders protection against radiation exposure in a whole organism model and the following issues were addressed using zebrafish, a non-hibernating vertebrate organism model: Does SA-like state, if induced prior to lethal doses of ionizing radiation exposure, confer any advantage or protection in terms of damage induction and survival? Does SA-like state mitigate and/or delays the manifestation of radiation-induced damage and lethality, when induced after ionizing radiation exposure?

Correspondence to: Dr. Indracanti Prem Kumar, Drug Repurposing \&Translational Research Lab, Institute of Nuclear Medicine and Allied Sciences, Brig SK Majumdar Road, Timarpur, Delhi, 110054, India, Tel: 91-11-23905301, 91-09958456999; Fax: 91-11-23919509; E-mail: prem_indra@yahoo.co.in

Key words: radiation, hypometabolic state, radiomitigation, zebrafish, suspended animation

Received: April 24, 2017; Accepted: May 26, 2017; Published: May 29, 2017 


\section{Materials and methods}

\section{Zebrafish maintenance and embryo collection}

Wild-type zebrafish (Danio rerio) were maintained following the standard protocol $[20,21]$. The fishes were kept at a room temperature of $28.5 \pm 2{ }^{\circ} \mathrm{C}$ with a $14 \mathrm{~h}$ light followed by $10 \mathrm{~h}$ dark cycle and fed twice a day with freshly hatched brine shrimp (Sanders, The Great Salt Lake, USA) and intermittent feeding with commercial flake food (AquadeneMicroPellet, China). Embryos from paired breeding were collected within $1 \mathrm{~h}$ of spawning, cleaned and grouped according to the stage of the development [22], and maintained in $90 \mathrm{~mm}$ petriplates in $1 \times \mathrm{E} 3$ medium ( $5 \mathrm{mM} \mathrm{NaCl}, 0.17 \mathrm{mM} \mathrm{KCl}, 0.33 \mathrm{mM} \mathrm{CaCl}_{2}, 0.33 \mathrm{mM} \mathrm{MgSO}_{4}$ ).

\section{Reagents and environmental condition}

All chemicals used for preparation of medium and buffers, unless otherwise indicated, were obtained from Sigma-Aldrich (St Louis, MO, USA). All experiments were carried out in accordance to the animal ethics guidelines of the Institute of Nuclear Medicine and Allied Sciences (INMAS), Delhi, India. Environmental Bio Bag Type A (Becton Dickinson and Co., MD, USA) were used for induction of anoxia following manufacturer's instructions. The onset of anoxia was determined by following the change in color with the resazurin indicator (pink to white) and in our hands, the color change indicating the onset of anoxia was observed within 30 minutes of sealing the bags. However, earlier studies have shown that small amount of oxygen remains on the water depending on the volume of the water, and around 60 to 90 minutes is required for achieving the complete anoxic state [23].

\section{Anoxia induced DH state, recovery and viability assessment}

Embryos at different developmental stages $(3,6,16,24,48,72$ and $96 \mathrm{hpf}$ ) were placed into the anoxia chamber in $4 \mathrm{ml}$ of $\mathrm{E} 3$ medium and maintained for 4 or $20 \mathrm{~h}$ at $28.5 \pm 2{ }^{\circ} \mathrm{C}$. After the stipulated time intervals, anoxia chambers were opened and embryos were transferred into normoxia E3 medium [24]. The embryos were observed for recovery and damage manifestations (pericardial edema, heartbeat, skin roughness and lethality) every 24 hours up to $144 \mathrm{hpf}$ or for the duration of the experiment. Embryos subjected to anoxia at $3-16 \mathrm{hpf}$ were assessed after they reached $24 \mathrm{hpf}$ stage while those at $24 \mathrm{hpf}$ and older were assessed within 1 hour after returning to normoxia basing on resuscitation of the heart-beat. The assessments for all groups of embryos were done until $144 \mathrm{hpf}$ including morphological endpoints like pericardial edema, heart rate, skin roughness and survival.

\section{Radiation and LD50}

To determine the optimal radiation dose for anoxia studies, embryos (3.3-3.8 hpf; $n=5$ per group) were exposed to different doses of gamma radiation at room temperature ( 5 to $40 \mathrm{~Gy}$ ) using a ${ }^{60} \mathrm{Co}$ source (Gamma cell 5000, Board of Radioisotope Technology, Mumbai, India; dose rate $1.12 \mathrm{KGy} / \mathrm{h}$ ). The assessments of developmental parameters, damage end points and lethality were monitored every 24 -hour until 144 hpf.

\section{Radioprotection and mitigation study}

For radioprotection protection studies, petri plates containing embryos $(n=6)$ at 3.5-3.8 hpf were placed inside the anoxia chambers, sealed and maintained for $20 \mathrm{~h}$ and exposed to $20 \mathrm{~Gy}$ gamma radiation. After 30 minutes of radiation exposure, the chambers were opened and embryos were transferred to normoxia medium and monitored for development, damage manifestations and death till $144 \mathrm{hpf}$. For radio mitigation studies, around 3.5 - 3.8 hpf staged embryos $(n=6)$ were exposed to $20 \mathrm{~Gy}$ and within 30 minutes, the embryos were transferred to anoxia chambers and maintained for 20 hours. Thereafter, the embryos were transferred to normoxia conditions, and the damage manifestations and lethality were monitored every 24 hours till $144 \mathrm{hpf}$.

\section{Assessment of heart rate, pericardial edema, micro-} ophthalmia, microcephaly and skin roughness

For assessing survival, beating heart was considered as the end point and percent survival was calculated as previously reported by Geiger et al [25]. Radioprotection or mitigation due to DH state was calculated by comparing the $\mathrm{DH}+$ radiation $(\mathrm{DH}+\mathrm{IR})$ group with the radiationalone group. Quantitative analysis of heart rate was done following the stopwatch method $[26,27]$. Briefly, embryos were anesthetized in $0.001 \%$ tricaine dissolved in $1 \times \mathrm{E} 3$ medium prior to counting under a microscope (Leica DMR, Germany) connected with a computer by an external camera (MDCE-5C, USB 2.0). The heartbeat of each embryo was counted for 1 minute using a stopwatch and expressed as an average of beats/minute of three individual experiments. $0.001 \%$ tricaine used for inducing anesthesia did not have any effect on heart rate of 24$144 \mathrm{hpf}$ staged embryos. The assessments of pericardial edema and micro-ophthalmia of embryos were analyzed by measuring the area of pericardium and eye following reported methods [28,29]. Briefly, embryos were anesthetized and placed laterally under the microscope, and images were captured every 24 hours till $144 \mathrm{hpf}$. The area measurements were done using a free hand measuring tool in Image J software (http://rsbweb.nih.gov/ij/) and expressed as the mean value of area (pixels) of three independent experiments. Similarly, for midbrain length, the distance between hindbrain and forebrain was measured at $48 \mathrm{hpf}$ and expressed as pixels of three independent experiments. Midbrain measurement was not done in older embryos (> $48 \mathrm{hpf}$ ) due to significant pigmentation and obscure brain boundaries. The skin roughness (SR) measurement was done by capturing the images of an initial somatic muscle portion behind the hindbrain and was assessed for the appearance of muscle fiber organization (+ve score), and disorganization (-ve score) was carried out. Based on +ve or -ve score the SR was expressed as \% of incidence of three independent experiments.

\section{Statistical analysis}

Results were presented as mean \pm S.D. of three individual experiments. Statistical significances between two independent groups were assessed by Student's t-test and one-way ANOVA followed with Fisher's post-hoc test using Microsoft Excel (MS Office 2007). A p-value $<0.05$ was considered significant.

\section{Results}

Reversibility of the suspended animation (SA)-like state in developing zebrafish embryos.

In our laboratory, induction of anoxia-mediated DH state was achieved within 2 hours of placing the embryos into the anoxic chamber. When embryos were brought back to the normoxia condition, the development resumed and with respect to control group, which were maintained in the normoxia environment, the development was found to be delayed by the duration for which the embryos were in the DH state (Figures 1A-1G). The reversibility of the DH state showed dependence on the duration for which the embryos were in SA-like state. Embryos at 3, 6, 16, 24, 48, 72 and 96 hpf when exposed to 20 hours of SA-like state exhibited a decreasing survival $(100,100,85,70$, 0,0 , and $0 \%$ respectively) (Figure $1 \mathrm{H}$ ). While late-staged embryos i.e 

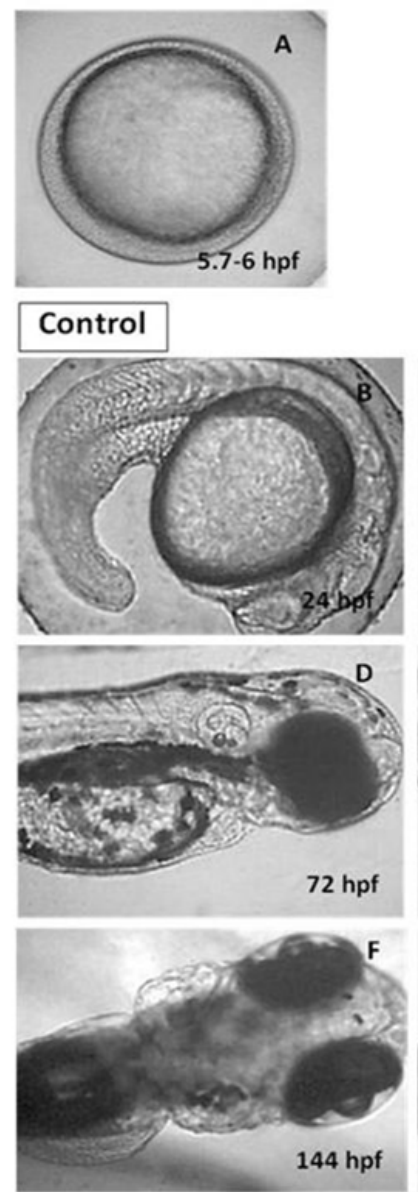

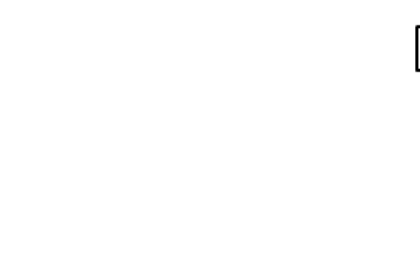

H
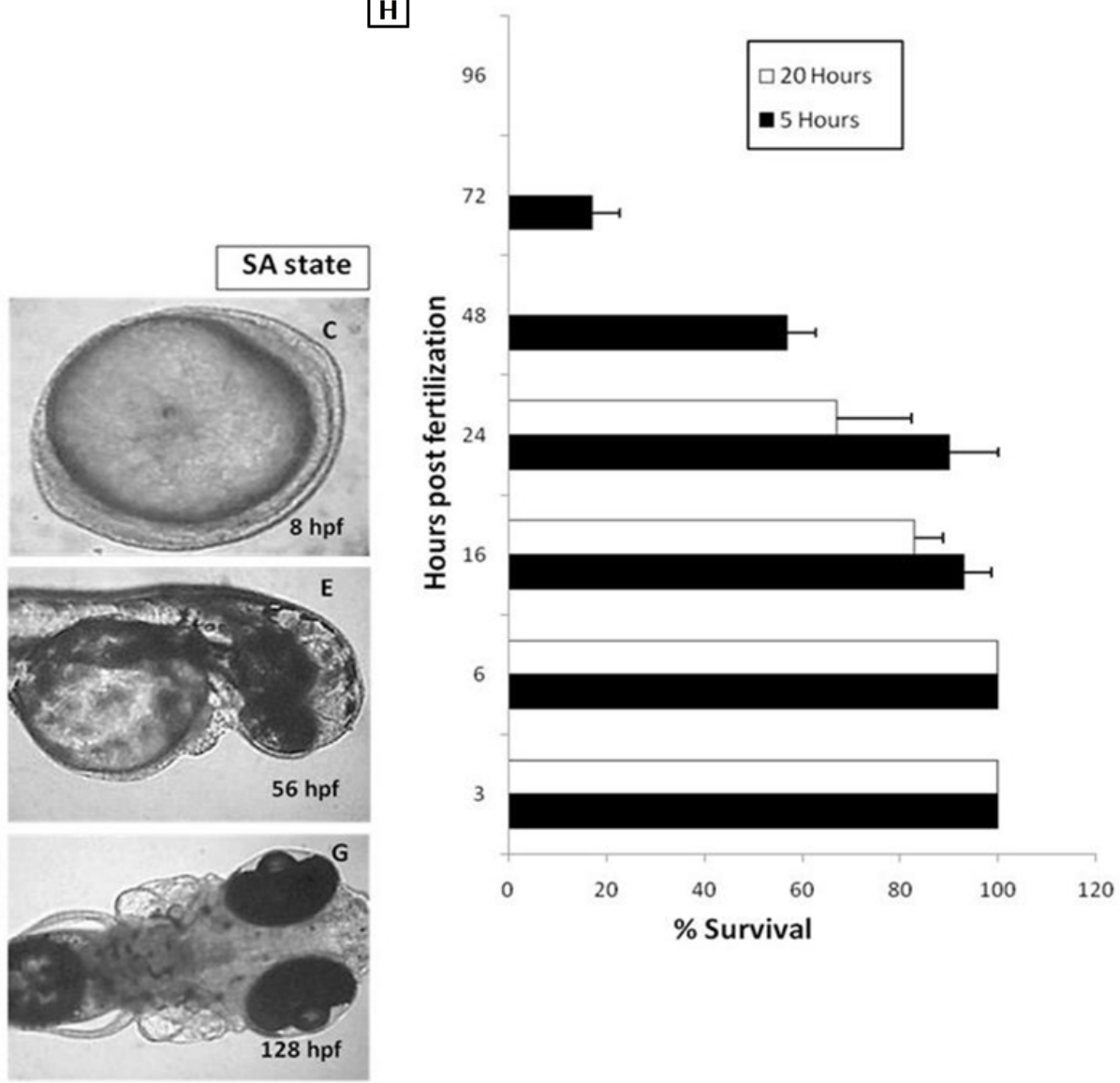

\% Survival

Figure 1. Effect of the anoxia-induced SA-like state on development of zebrafish embryos. $5.7 \mathrm{hpf}$ zebrafish embryos were put to anoxic environment for a duration of 18 hours and thereafter shifted to normoxic medium and further development was observed at different time points. $5.7 \mathrm{hpf}$ zebrafish embryos allowed to develop in normoxic environment were used as a control group. Panel A shows a representative image of 5.7-6 hpf old zebrafish embryo. Panels B, D and F show the representative images of developing zebrafish embryos in normoxic conditions after 24, 48 and 72 hours respectively. Panels C, E and G show the representative images of developing zebrafish embryos in normoxic conditions after 24,48 and 72 hours respectively. Note that the developing embryos put to SA-like state were lagging behind, in development, by 16 hours (based on the head-to-trunk angle, data not shown). Panel H shows a survival histogram of different stages of embryos with different SA state durations. All images are representative of three independent experiments. Each value represents the mean $\pm \mathrm{SD}, \mathrm{n}=10$, $\mathrm{p}<$ 0.003 ; between two SA-like state groups.

older than 48 hpf could not tolerate even 5 hours of the DH-like state showing $30 \%$ survival at $72 \mathrm{hpf}$ and $0 \%$ at $96 \mathrm{hpf}$ (Figure $1 \mathrm{H}$ ).

\section{Effects of ionizing radiation on zebrafish embryos}

Survival: Exposure of 3.5-3.8 hpf embryos to different doses of ionizing radiation resulted in a dose-dependent damage induction and lethality (Figure 2). Morphological assessment of the embryos exposed to increasing doses of radiation showed an increasing overall damage manifestation and deterioration (Figure 3A). The lowest dose (5 Gy) was used for this study to induce minimal effects while deaths in case of higher radiation doses were more pronounced. The survival after 144 hours was $80,70,20 \%$ for 5, 10, $20 \mathrm{~Gy}$, respectively. The exposure of the even higher doses, resulted in death of the embryos within 72 hours (Figure 2B).

Heart rate: The heart rate (beats/minute) of untreated control embryos initially increased from 120 beat/minimum at $24 \mathrm{hpf}$ to $160 \mathrm{beat} / \mathrm{minimum}$ at $48 \mathrm{hpf}$ and thereafter remained plateau at the end of experiment (Figure 3C). Exposure to 5 and 10 Gy radiation did not change this pattern until $72 \mathrm{hpf}$. However, unlike untreated embryos, after $72 \mathrm{hpf}$, the IR embryos exhibited a significantly reduced heartbeats (140 beats per minute in comparison to 160 beats for un-

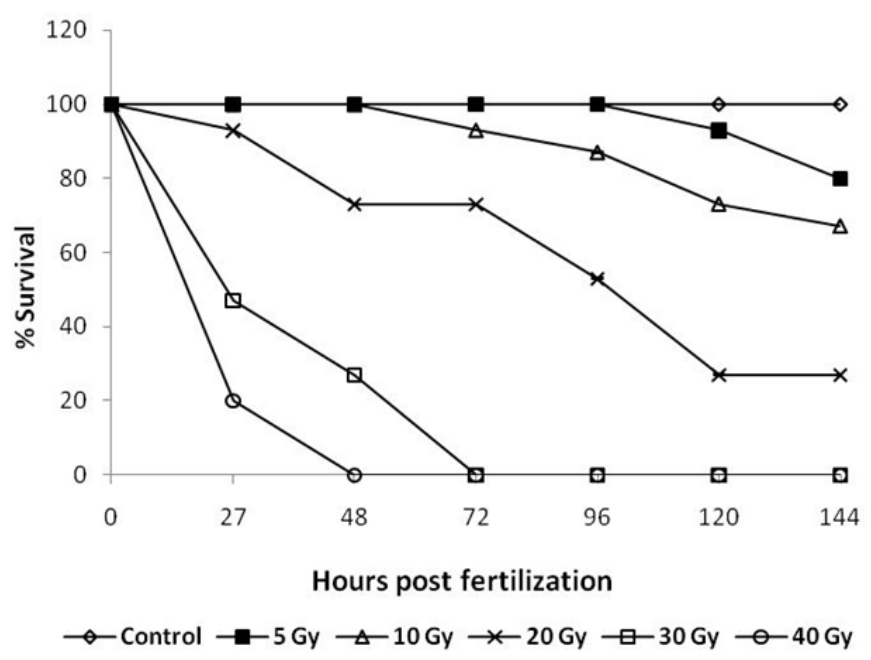

Figure 2. Radiation dose response of developing zebrafish. Groups of 5.7-6 hpf old zebrafish embryos were exposed different doses of gamma radiation, and survival were assessed at indicated time points post radiation exposure. Data presented is from three independent experiments. Each value represents the mean $\pm S D, n=6$. $P<003$; versus nonirradiated control group. 
irradiated embryos). Embryos exposed to 20 Gy perturbed the normal heartbeat pattern, and the heartbeat remained at around 100 beats per minute until 96 hours, which thereafter showed a steep decline and was found to be 60 beats per minute in the surviving embryos after $144 \mathrm{hpf}$. Higher doses (30 and 40 Gy) showed more pronounced effects, and a steep decline were observed, and heartbeat was undetectable after 48 and $72 \mathrm{hpf}$ for 30 and 40 Gy treated embryos respectively (Figure 3C).

Eye size \& midbrain length: Untreated zebrafish embryos have shown a normal increase in the size of the eye till $72 \mathrm{hpf}$ and thereafter a plateau was observed until $144 \mathrm{hpf}$ (Figure 3D). A similar trend was observed in case of embryos exposed to 5 and $10 \mathrm{~Gy}$. However, $20 \mathrm{~Gy}$ exposure led to reduced eye development until $96 \mathrm{hpf}$ and thereafter there were significant degeneration and decrease in the size was observed until 144 hpf (Figure 3D). In case of higher doses (>20 Gy), the size of the eye has shown a steep decline from $24 \mathrm{hpf}$ onwards, and the trend was continued until their deaths (Figure 3D). Similarly, the midbrain length at $48 \mathrm{hpf}$ was not markedly affected by 5 Gy and 10 Gy (Figure 3B). However, the 20 Gy exposure has led to a significant decrease in the size of the midbrain length which was found to be profound in case of $30 \mathrm{~Gy}$. The effect of $40 \mathrm{~Gy}$ could not be assessed as the embryos were dead by $48 \mathrm{hpf}$ (Figure 3B).

Pericardial edema (PE): Radiation in a dose-dependent fashion increased the pericardial edema formation. Exposure to $20 \mathrm{~Gy}$ resulted in an increase in the volume of pericardial sac, beginning at $48 \mathrm{hpf}$, which continued to expand during the entire duration of experiment (till $144 \mathrm{hpf}$ ). While the $40 \mathrm{~Gy}$ exposure led to appearance of pericardial edema at 27 hours and continued to increase till their deaths ( $48 \mathrm{hpf}$ ). Lower doses $(<20 \mathrm{~Gy})$ did not induce any significant increase in the pericardial area during the entire duration when compared to untreated control (Figure 3E).

\section{Effect of DH state on radiation induced damage and lethality}

From the dose-response studies, 20 Gy (LD 80/6) was chosen for further radioprotection and mitigation studies. Embryos in the $\mathrm{DH}$ state (for $20 \mathrm{~h}$ ) exposed to $20 \mathrm{~Gy}$ ionizing radiation (hereafter expressed as DH + IR) exhibited significant protection when compared
A

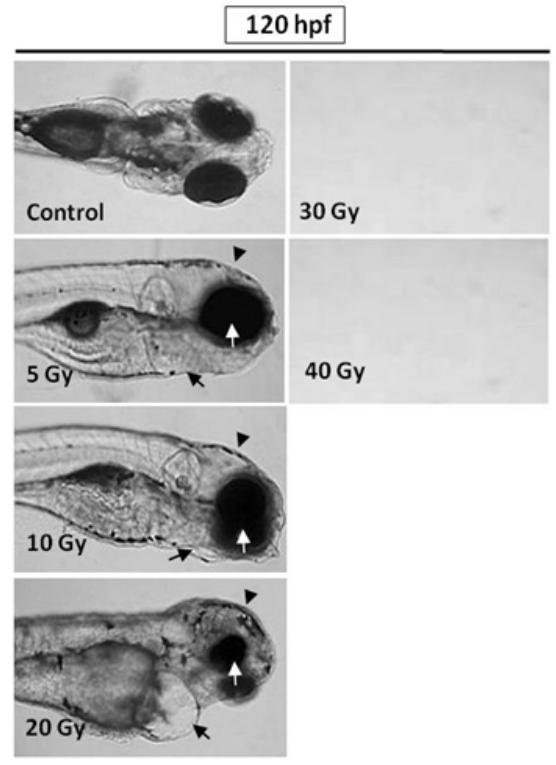

B

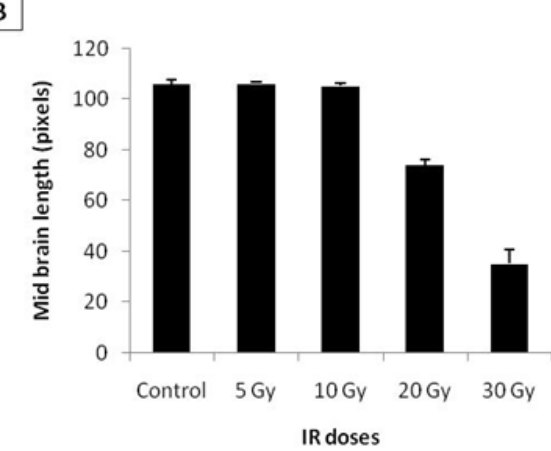

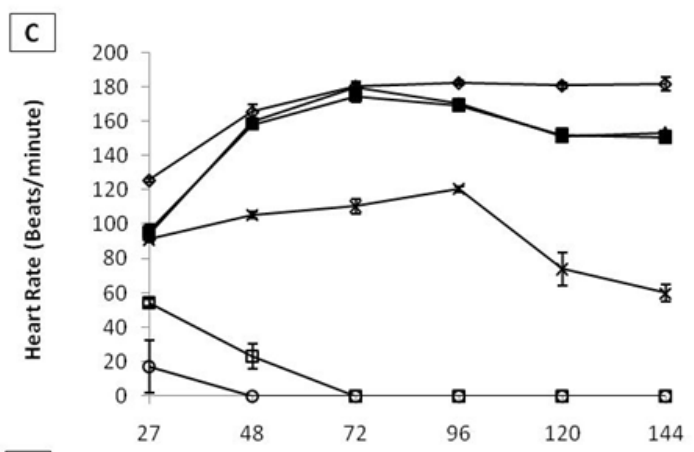

D

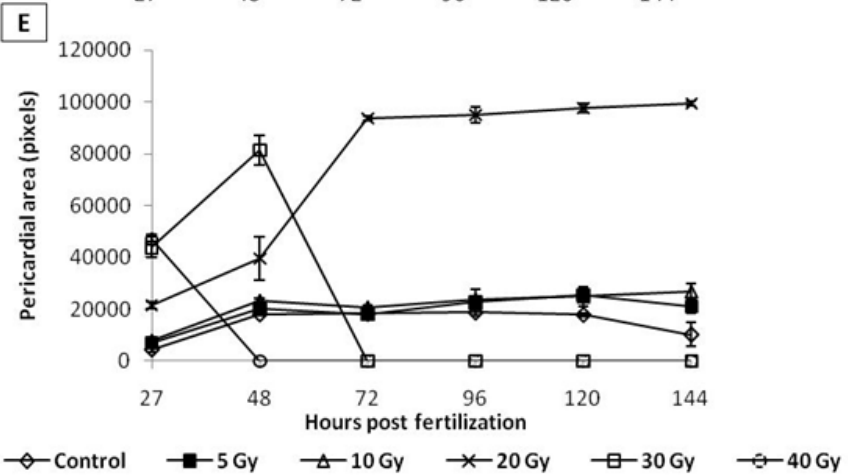

E

Figure 3. Effect of different doses of IR on heart rate, pericardial edema, microcephaly and micro-opthalmia in zebrafish embryos. Panel A shows representative images of embryos exposed to different IR doses. Black arrows, arrowhead and white arrows indicate pericardial edema, microcephaly and micro-opthalmia respectively). B shows the effect of different doses of IR on microcephaly, expressed as midbrain length in pixels at $48 \mathrm{hpf}$ (measured at a lateral view). C Changes in heart rate with increasing doses of IR and expressed as beats per minute. D and $\mathrm{E}$ show effect of different doses of IR on micro-opthalmia and pericardial edema. Each value represents the mean $\pm \mathrm{SD}$, $\mathrm{n}=5$ of at least three independent experiments. 
to the radiation-alone group and rendered $80 \%$ survival in comparison to $20 \%$ survival in the 20 Gy-irradiated group on 6th post irradiation day (Figure 4). The surviving embryos have exhibited very little damage manifestation and continued to reach adulthood without any gross deformities. The DH state significantly rescued the embryos from radiation-induced damage manifestations studied (heart rate, eye size, pericardial edema and midbrain length) (Figure 5). Embryos in the DH + IR group showed significant protection against radiation-induced reduction in heart rate. Upon transfer to normoxia conditions, there was an initial increase in the heart rate till $72 \mathrm{hpf}$ which thereafter remained plateau till $144 \mathrm{hpf}$ (Figure 5A). A similar trend was also observed in the case of untreated embryos (Figure 5A). Similarly, the $\mathrm{DH}$ state reduced radiation-induced pericardial edema formation and exhibited a pericardial volume similar to that observed in the case of the control group during the entire experimental duration (Figure 5B). The DH state significantly protected eye and brain from radiationinduced damage and degradation and upon transfer to normoxia conditions, both eye and brain showing a developmental pattern similar to the untreated control group. While $20 \mathrm{~Gy}$ exposure lead to a significant reduction of eye and brain development and an increase in the opaqueness which persisted till $144 \mathrm{hpf}$ (Figures 5C, 5D). The midbrain lengths in the cases of control and 20 Gy exposed groups were found to be $105 \pm 0.8$ and $72 \pm 2.9$ pixels, respectively, while the $\mathrm{DH}+\mathrm{IR}$ group has shown an average midbrain length of $104 \pm 2$ pixels. As observed in case of other end points, the $\mathrm{DH}$ state protected the embryos from radiation-induced muscle fiber degradation (Figure 6). Radiation-induced gross changes in the organization of muscle fibers and subsequently led to skin disruption at $27 \mathrm{hpf}$ and by $96 \mathrm{hpf}$, and all the irradiated embryos have shown optimal skin disruption characteristic (Figure 6C). The DH state significantly reduced the incidence of skin disruption (only 10\% of the embryos exhibited skin deformities) (Figure 6D).

\section{DH state mitigates radiation-induced damage and lethality}

To address the question of whether an induction of DH state after irradiation (hereafter referred as IR $+\mathrm{DH}$ ) delays ionizing radiationinduced damage manifestation and lethality, embryos were exposed (20 Gy) at 3.5-3.8 hpf and immediately the DH state was induced for a duration of $20 \mathrm{~h}$. When transferred to normoxic conditions the embryos have shown a delay in development (by 18h) and were found to be at $5.7 \mathrm{hpf}$ stage and resumed development while the corresponding untreated control or irradiated group were found to be at $24 \mathrm{hpf}$ stage (Figure 7C, 7D). The DH state mitigated radiation-induced lethality and exhibited a survival advantage of $34 \%$ over the radiation control (Figure $7 \mathrm{~K}$ ). The heart rate in the surviving embryos in the IR + DH group was found to be significantly higher than $20 \mathrm{~Gy}$ exposed embryos during the entire duration of experiment (Figure 8A). The volume of pericardial edema in surviving embryos of the IR + DH group $(85 \pm$ 1.3) was also found to be significantly less than 20 Gy-exposed embryos (70 \pm 2.8$)$. Similar protection was also observed with midbrain length (Figure 8C). Eye area of irradiated embryos began to increase with a slow rate from 27 to $144 \mathrm{hpf}$ whereas in the IR $+\mathrm{DH}$ group, the eye area increased significantly from 48 to $72 \mathrm{hpf}$ and then declined. But in control embryos, there was a constant increase in the area from 27 to $144 \mathrm{hpf}$ (Figure 8D). However, these results indicate that the radiation damage progress in the eye was delayed by $24 \mathrm{~h}$. The muscle damage perturbation delay and amelioration by the SA- like state are illustrated in Figure 9. It was found that muscle fiber appearance and organization were similar as our previous observations in irradiated embryos. However, the severity was less in the IR + DH group (Figures
9A-D). The number of incidence of skin roughness began to increase at $27 \mathrm{hpf}$, and at $72 \mathrm{hpf}$ it reached $100 \%$ in irradiated embryos, whereas it began to increase at $72 \mathrm{hpf}$ and reached $100 \%$ at $144 \mathrm{hpf}$ in the IR + DH group (Figures 9E).

\section{Discussion}

Reversible hypometabolic states or DH states have been well studied in a number of organisms and are known by a variety of terms, including hibernation, aestivation and torpor [7]. The present study investigated the radioprotective and mitigating effects of the SA-like state in zebrafish embryos. The embryos exhibited an inverse relationship between the age and reversibility of the SA-like state, which is in corroboration with earlier reports [24,25]. Anoxia effectively induced a hypometabolic state as was evident to the halting of development and slowing down of the heartbeat which strongly suggests the extremity of hypometabolic state. In fact, diminishing heart rate is a reliable end point for assessing the hypometabolic state. Systematic evaluation of morphological manifestation like pericardial edema, skin roughness and bradycardia suggests that zebrafish embryos up to $24 \mathrm{hpf}$ successfully survived 16 hours of anoxia without any apparent damages. The successful survivals of the anoxic condition by early staged embryos can be explained by the fact that prior to development of circulation, embryos have the ability to adapt to anoxic conditions and halt the development. Further, it has been reported that those organisms which lack functional circulating system are more efficient in adapting to anoxic conditions [30]. However, the inability to survive longer durations of $\mathrm{DH}$ state in case of latestage embryos could be attributed to the presence of the functional circulator system where quick sensing and adaptability are not effective. Though the late-stage embryos did survive shorter durations (hours), but the surviving embryos exhibited damage manifestation when brought back to normoxia conditions, which could be due to free radical generation resulting from reperfusion of oxygen [31]. Among different end points studied, it is observed that embryos exposed to anoxia exhibited slowing down of heart rate, which could be due to the reduced metabolism [25]. Anoxia-induced DH states have already been shown to render protection against different stresses; hence it was interesting to investigate its possible radioprotective action. In view of this, a radiation dose-response study was undertaken with the most radiosensitive stage, i.e. mid-blastula transition state. 20 Gy was found to elicit LD80 in 6 days time, and all further studies were undertaken with this LD80 dose. The results clearly suggested that the DH state offered significant protection against radiation-induced organ damage and lethality. One of the possible mechanisms could be the absence or minimal presence of molecular oxygen in the system as oxygen is well understood to play a major role in generation of oxygen centered radicals [1]. Indeed, studies with cell lines under different oxygen tensions have clearly reported that low oxygen tensions lead to significant radioprotection. DH state when introduced after exposure to radiation resulted in delaying damage manifestation clearly suggesting that low metabolic state was indeed capable of delaying the radiation damage manifestation. The marginal mitigation by the $\mathrm{DH}$ state could be attributed to the fact that the lack of oxygen in the post-irradiation period might be reducing the secondary radical generation which is well known to contribute towards overall damage manifestation. Present study clearly suggests that a reversible hypometabolic state is achievable in vertebrates and renders significant protection against radiation-induced lethality. Anoxia was used as a method of choice for this study due to ease, however, similar approach may not be feasible for higher mammals and for human application. Nevertheless, 

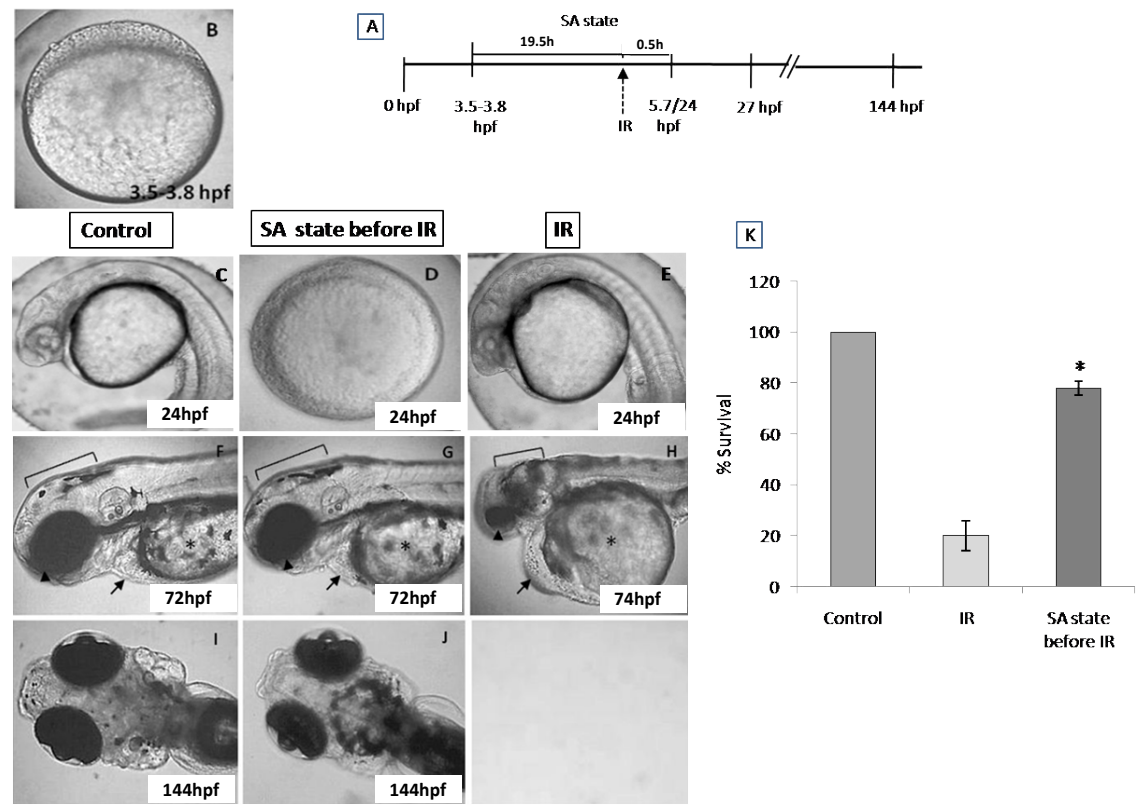

Figure 4. SA-like state attenuates the radiation-induced lethality in zebrafish embryos. Group of ( 3.8 hpf staged) zebrafish embryos were exposed to ionizing radiation in normoxic environment or when they are in SA-like state and 30 minutes later, the embryos were shifted to normoxic environment, and mortality was till 6 post irradiation days (144hpf). Figures C.F.I represents the un-irradiated control embryos imaged at 24, 72 and 144 hpf. Figures D, G, J represents zebrafish embryos exposed to IR while in SA-like state and imaged at 24,72 and $144 \mathrm{hpf}$. Note the delayed embryonic development in the SA-like state group exposed to IR. E, H are the representative images of developing embryos exposed to IR while in normoxic environment. Qualitative comparisons of morphologic aberration, microcephaly, micro-ophthalmia, pericardial edema and failure of yolk sac absorption are indicated by bracket, arrowhead, arrow and asterisk respectively. Figure K. Histogram showing the survival of zebrafish observed $6^{\text {th }}$ day-after different treatments. All images are representative of three independent experiments. Each value represents the mean $\pm \mathrm{SD}, \mathrm{n}=6 .{ }^{*}, \mathrm{p}<0.003$. Comparisons were made between IR and IR+SA-like state group.
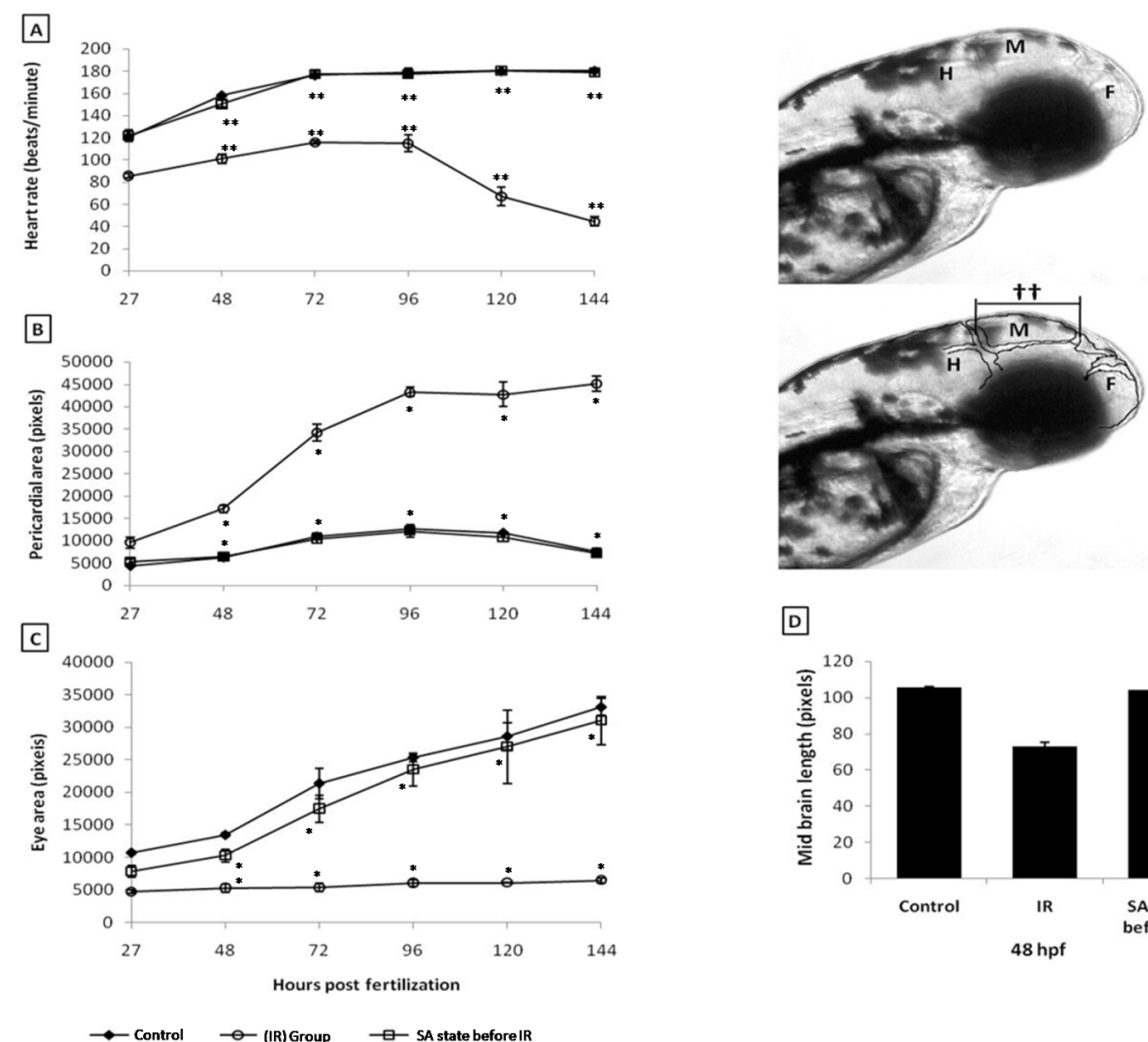

四

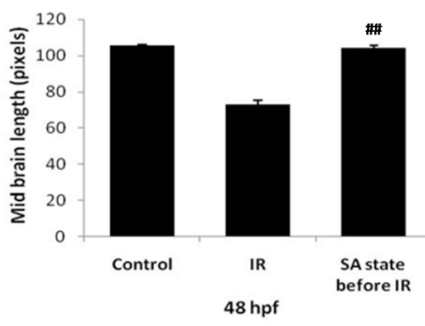

Figure 5. SA-like state protects zebrafish from radiation induced reduction in heart rate, pericardial edema, micro-ophthalmia and midbrain length. A. Changes in heart rate measured at different indicated time points after different treatments. B and C. Changes in pericardial sac volume and eye size measured at different indicated time points after different treatments. D. Microcephaly, expressed as the measurement of midbrain length in pixels at $48 \mathrm{hpf}$. Inset images above the panel $\mathrm{D}$ illustrate the brain regions $(\mathrm{HB}=\mathrm{HindBrain}, \mathrm{MB}=\mathrm{MidBrain}$ and $\mathrm{FB}=$ ForeBrain) and the length of the midbrain indicated as symbol $(\dagger \dagger)$. Each value represents mean $\pm \mathrm{SD}, \mathrm{n}=6$ of at least three independent experiments. $*, \mathrm{p}<005, * *, \mathrm{p}<003 \# \#, \mathrm{p}<003$. Comparisons were made between untreated control and IR group or between IR and SA-like state +IR group. 


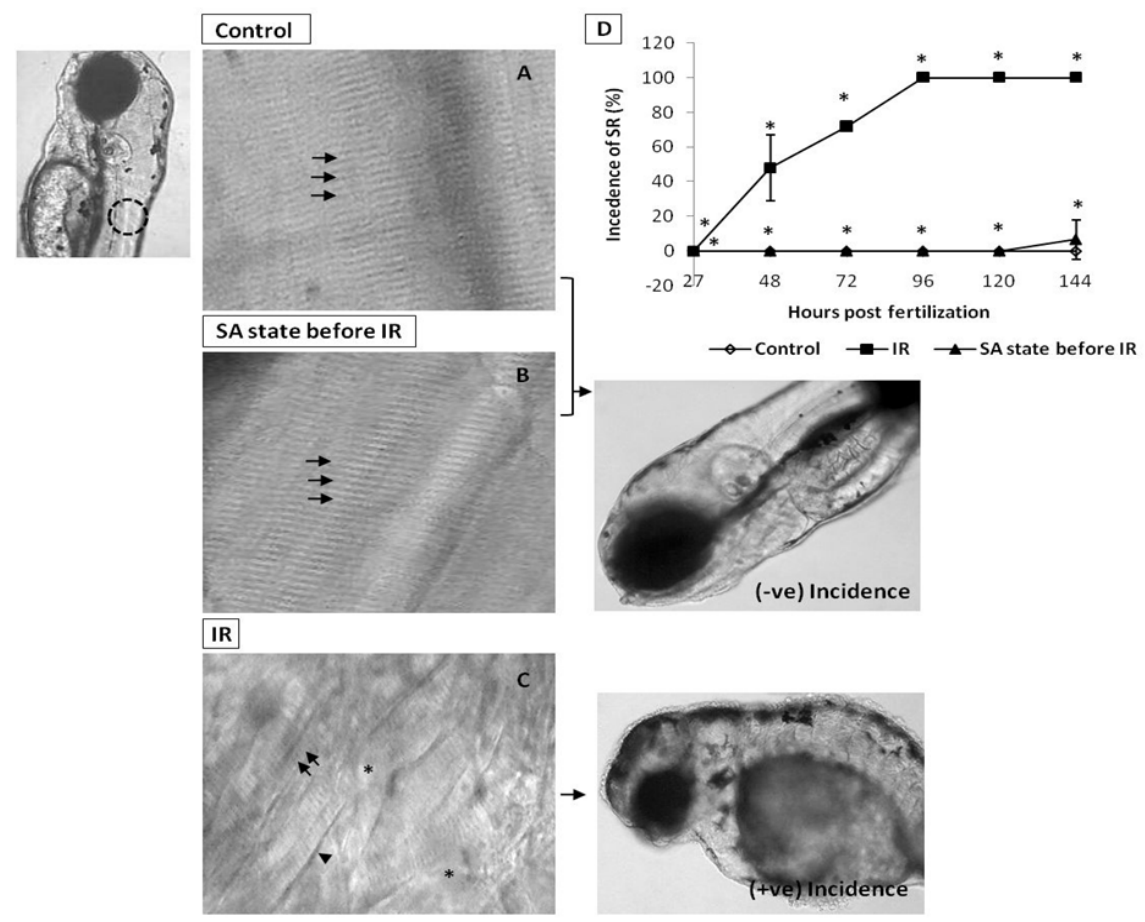

Figure 6. SA-like state attenuates IR-induced skin damages. Panel A shows muscle fibers (indicated by arrow) of the non-irradiated control embryos at 120 hpf. Image, left of panel A illustrates the somitic region (by dashed circle) used for skin analysis. B shows attenuation of IR effect (at $120 \mathrm{hpf}$ ) on muscle fibers (indicated by arrows) in zebrafish embryos exposed to IR while in SA-like state. Panel C shows disrupted muscle fiber organization with disappearing and irregular prominent solid line (indicated by arrow's asterisk and arrowhead respectively) in IR embryo at 120 hpf. Images at the right side of panel B and C illustrate the negative and positive incidence of skin roughness (SR). D shows the incidence of SR(\%) zebrafish with varied treatment at indicated time points. Each value represents mean $\pm \mathrm{SD}, \mathrm{n}=6 .{ }^{*}, \mathrm{p}<005$. Comparisons were made between untreated control and IR group or between IR and IR+SA-like state group.
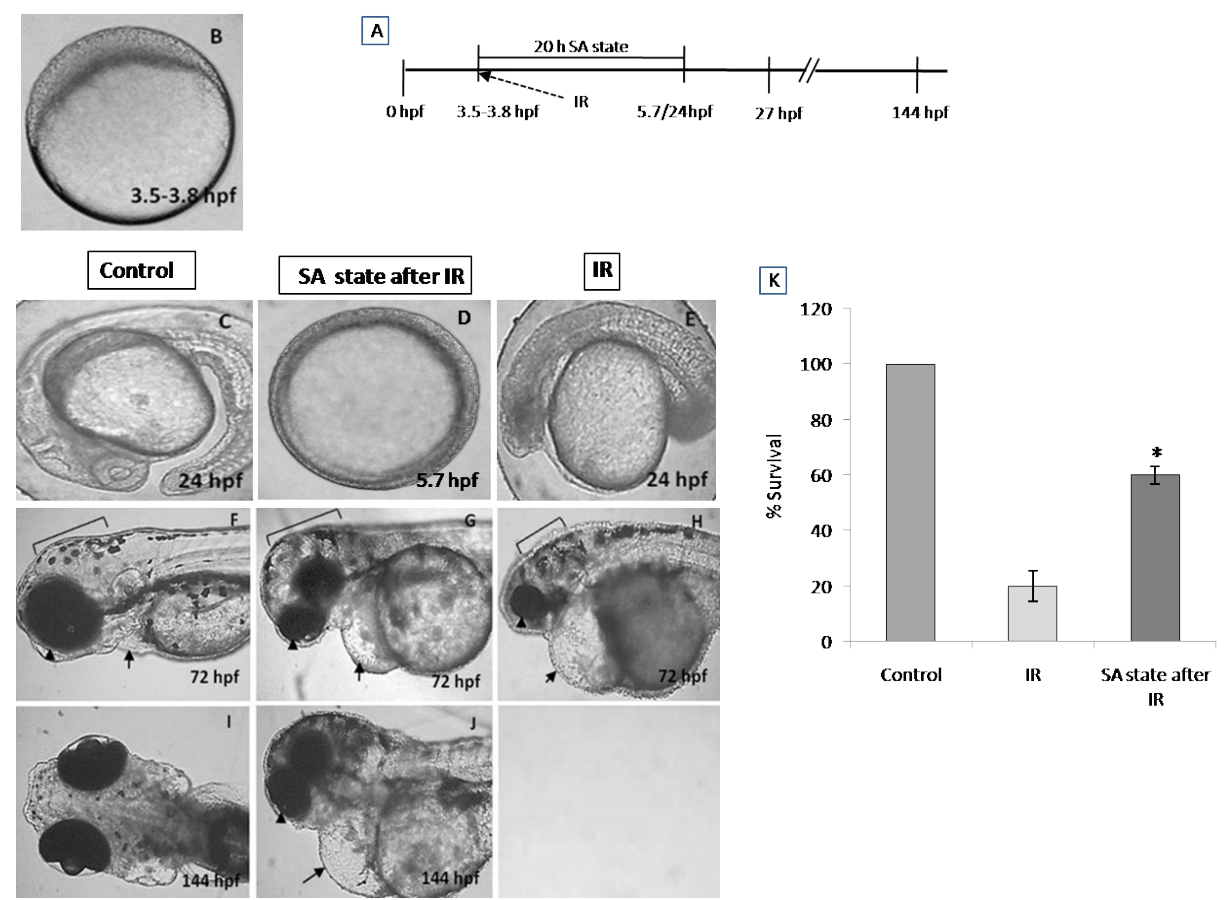

IR

Figure 7. SA-like state mitigates radiation-induced lethality in zebrafish embryos. Panel A shows a schematic representation of experimental setup. Group of (3.8 hpf staged; Figure A) zebrafish embryos were exposed to ionizing radiation in normoxic environment and subsequently they were put to SA-like state for 20 hours. Thereafter the embryos were shifted to normoxic environment and mortality was till 6 post irradiation days (144hpf). Figures C.F.I represents the un-irradiated control embryos imaged at 24 , 72 and 144 hpf. Figures D, G, J represents zebrafish embryos where SA-like state was induced post irradiation and imaged at 24,72 or 144 hpf. Note the delayed embryonic development in SA-like state group exposed to IR. E, H are the representative images of developing embryos exposed to IR while in normoxic environment. Qualitative comparison of morphologic aberration like microcephaly, micro-ophthalmia and pericardial edema are indicated by bracket, arrowhead and arrow respectively. Figure K. Histogram showing the survival of zebrafish observed $6^{\text {th }}$ day after different treatments. All images are representative of three independent experiments. Each value represents mean $\pm \mathrm{SD}, \mathrm{n}=6 .{ }^{*}, \mathrm{p}<0.003$. Comparisons were made between untreated control and IR group or between IR and IR+SA-like state group. 

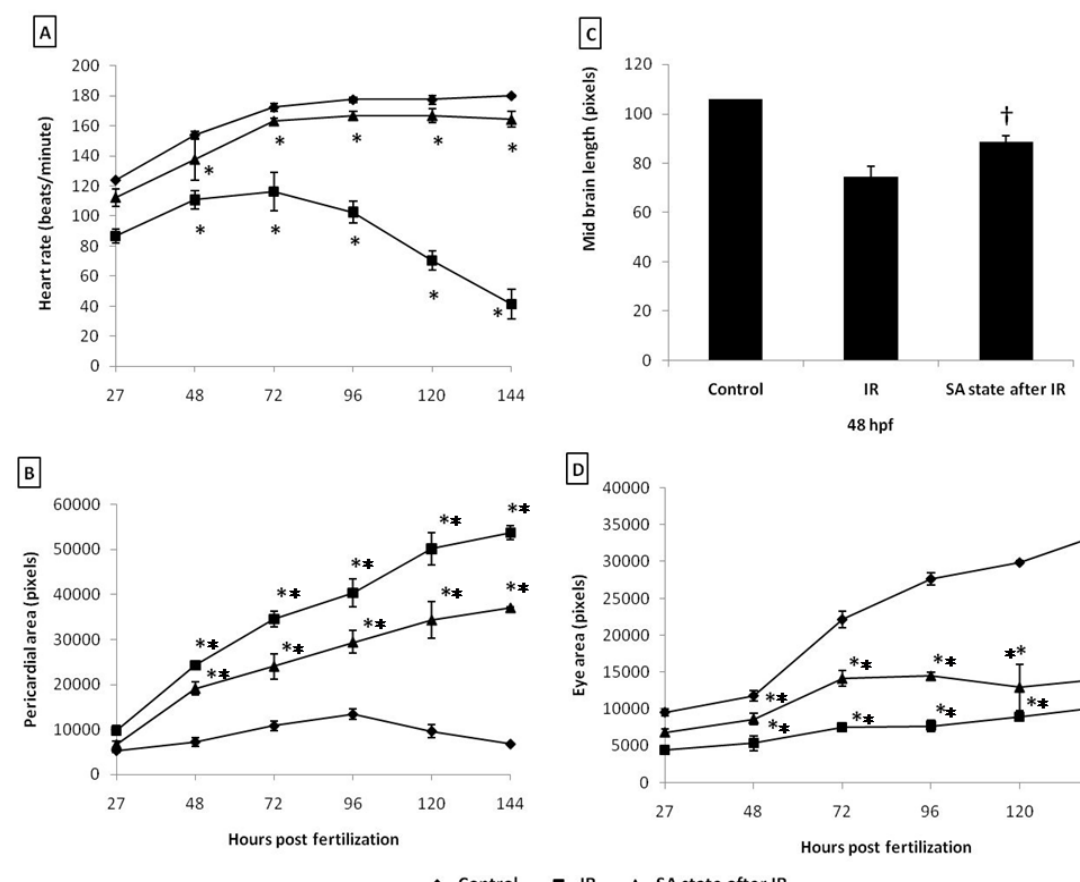

D 40000

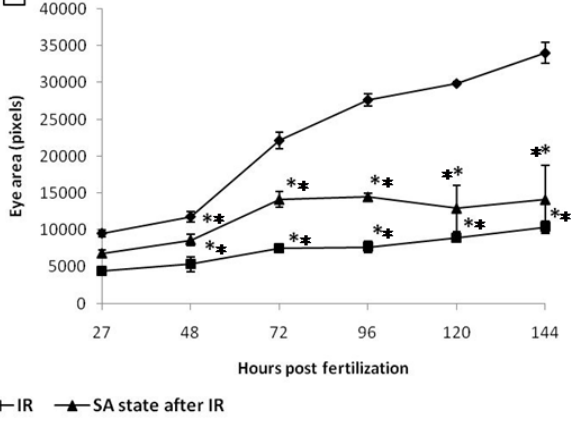

Figure 8. SA-like state mitigates radiation induced reduction in heart rate, pericardial edema, micro-ophthalmia and midbrain length in zebrafish embryos. Each error bar is $\pm \mathrm{SD}, \mathrm{n}=6$ of at least three independent experiments. Each value represents mean $\pm \mathrm{SD}, \mathrm{n}=6$ of at least three independent experiments. $*, \mathrm{p}<005, * *, \mathrm{p}<003, \dagger, \mathrm{p}<003$; Comparisons were made between untreated control and IR group or between IR and IR+SA-like state group.

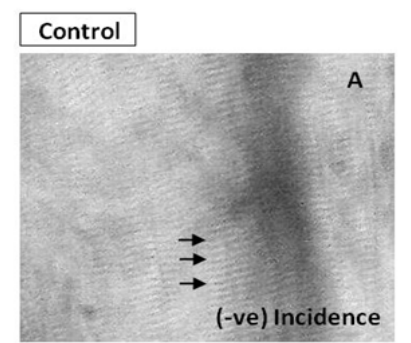

IR
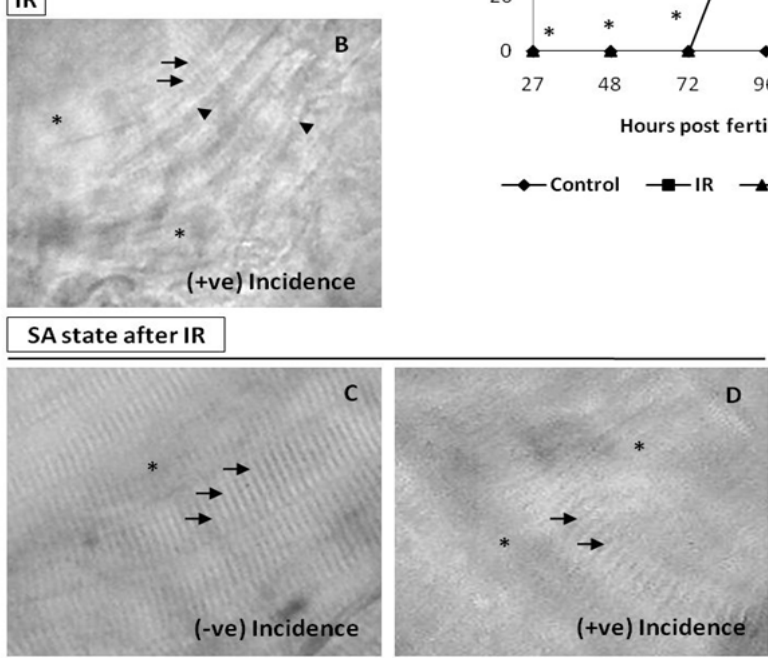

Figure 9. SA-like state mitigates IR-induced skin damage. A. Representative image ( $120 \mathrm{hpf}$ ) of intact muscle fibers (indicated by arrows) in non-irradiated control embryos B. Representative image of skin showing the effects of IR. The disrupted muscle fiber organization (indicated by arrows), disappearing and irregular prominent solid line (indicated by asterisk and arrowhead respectively) in IR embryo at $120 \mathrm{hpf}$. C. Image showing the mitigative effect of SA-like state when induced after IR (imaged at $120 \mathrm{hpf}$ ) on muscle fiber organization (indicated by arrows) or their disappearance (indicated by asterisk) in zebrafish embryos. The embryos with these features were counted as negative incidence. D. Representative image of embryos which were considered positive incidence. Note that different damage end points are evident. E. Percentage of incidence of SR(\%) zebrafish with varied treatment at indicated time points. Each value represents mean $\pm \mathrm{SD}, \mathrm{n}=6$. $^{*}, \mathrm{p}<005$. Comparisons were made between untreated control and IR group or between IR and IR+SA-like state group. 
in view of the significant benefits the hypometabolic state can offer to the management of radiation-induced damage, further studies are warranted in mammals using pharmacologically induced reversible hypometabolic states.

\section{References}

1. Hall E, Giaccia A (2006) Radiobiology for the Radiologist. Lippincott Williams \& Wilkins, Philadelphia, USA.

2. Greenberger JS (2009) Radioprotection. In Vivo 23: 323-336. [Crossref]

3. Riley PA (1994) Free radicals in biology: oxidative stress and the effects of ionizing radiation. Int J RadiatBiol65: 27-33. [Crossref]

4. Rubin P, Casarett GW (1968) Clinical Radiation Pathology Saunders, WB, Philadelphia, USA.

5. Baird BJ, Dickey JS, Nakamura AJ, Redon CE, Parekh P, et al. (2011) Hypothermia postpones DNA damage repair in Irradiated cells and protects against cell killing. Mutat Res 711:142-149. [Crossref]

6. Prasad KN (1995) Handbook of radiobiology. Boca Raton, FL, USA.

7. Melvin RG, Andrews MT (2009) Torpor induction in mammals: Recent discoveries fueling New ideas Trends. Endocrinol Metab 20:490-498. [Crossref]

8. Wang L, Wolowyky M (1998) Torpor in mammals and birds. Can J Zool 66:133-137.

9. Andrews MT (2007) Advances in molecular biology of hibernation in mammals. Bioessays 29: 431-440. [Crossref]

10. Blackstone E, Morrison M, Roth MB (2005) H2S induces a suspended animation-like state in mice. Science 308: 518. [Crossref]

11. Beltowski J (2007) Hydrogen sulfide (H2S): the new member of gasotransmitter. Family Biomedical Rev 18:75-83.

12. Aslami H, Heinen A, Roelofs JJ, Zuurbier CJ, Schultz MJ, et al. (2010) Suspended animation inducer hydrogen sulfide is protective in an in vivo model of ventilatorinduced lung injury. Intensive Care Med 36:1946-1952. [Crossref]

13. Aslami H, Schultz MJ, Juffermans NP (2009) Potential applications of hydrogen sulfide-induced suspended animation. Curr Med Chem 16: 1295-1303. [Crossref]

14. Szabó C (2007) Hydrogen sulphide and its therapeutic potential. Nat Rev Drug Discov6: 917-935. [Crossref]

15. Miller DL, Roth MB (2007) Hydrogen sulfide increases thermotolerance and lifespan in Caenorhabditis elegans. Proc Natl Acad Sci U S A 104: 20618-20622. [Crossref]

16. Pereira TC, Lopes-Cendes I (2009) Cryptic anhydrobiotic potential in man: implications in medicine. Med Hypotheses 73: 506-507. [Crossref]
17. Young JD, Taylor E (1998) Meditation as a Voluntary Hypometabolic State of Biological Estivation. News Physiol Sci 13: 149-153. [Crossref]

18. McAleer MF, Davidson C, Davidson WR, Yentzer B, Farber SA, et al. (2005) Novel use of zebrafish as a vertebrate model to screen radiation protectors and sensitizers. Int J RadiatOncolBiol Phys 61:10-13. [Crossref]

19. Davidson W, Ren Q, Kari G, Kashi O, Dicker AP, et al. (2008) Inhibition of p73 function by Pifithrin-alpha as revealed by studies in zebrafish embryos. Cell Cycle 7 : 1224-1230. [Crossref]

20. Westerfield M (1995) The Zebrafish Book: A guide for laboratory use of zebrafish Danio Rerio. University of Oregon Press. Eugene, OR, USA.

21. Sanders GE1 (2012) Zebrafish housing, husbandry, health, and care: IACUC considerations. ILAR J 53: 205-207. [Crossref]

22. Kimmel CB1, Ballard WW, Kimmel SR, Ullmann B, Schilling TF (1995) Stages of embryonic development of the zebrafish. Dev Dyn203: 253-310. [Crossref]

23. Padilla PA, Roth MB (2001) Oxygen deprivation causes suspended animation in the zebrafish embryo. Proc Natl Acad Sci U S A 98: 7331-7335. [Crossref]

24. Mendelsohn BA, Kassebaum BL, Gitlin JD (2008) The zebrafish embryo as a dynamic model of anoxia tolerance. Dev Dyn237: 1780-1788. [Crossref]

25. Geiger GA, Parker SE, Beothy AP, Tucker JA, Mullins MC, et al. (2006) Zebrafish as a "biosensor"? Effects of ionizing radiation and amifostine on embryonic viability and development. Cancer Res 66: 8172-8181. [Crossref]

26. Zhu X, Zhu L, Li Y, Duan Z, Chen W, et al. (2007) Developmental toxicity in zebrafish (Danio rerio) embryos after exposure to manufactured nanomaterials: buckminsterfullerene aggregates (nC60) and fullerol. Environ ToxicolChem26: 976979. [Crossref]

27. Abdelkader TS, Chang SN, Kim TH, Song J, Kim DS, et al. (2013) Exposure time to caffeine affects heartbeat and cell damage-related gene expression of zebrafish Danio rerio embryos at early developmental stages. J ApplToxicol33: 1277-1283. [Crossref]

28. Teraoka H, Dong W, Tsujimoto Y, Iwasa H, Endoh D, et al. (2003) Induction of cytochrome P450 1A is required for circulation failure and edema by 2,3,7,8-tetrachlorodibenzo-p-dioxin in zebrafish. BiochemBiophys Res Commun304: 223-228. [Crossref]

29. Kashyap B, Frederickson LC, Stenkamp DL (2007) Mechanisms for persistent microphthalmia following ethanol exposure during retinal neurogenesis in zebrafish embryos. Vis Neurosci24: 409-421. [Crossref]

30. Bakkers J (2011) Zebrafish as a model to study cardiac development and human cardiac disease. Cardiovasc Res 91: 279-288. [Crossref]

31. Zweier JL, Flaherty JT, Weisfeldt ML (1987) Direct measurement of free radica generation following reperfusion of ischemic myocardium. Proc Natl Acad Sci U S A 84: 1404-1407. [Crossref]

Copyright: (C2017 Ghosh S. This is an open-access article distributed under the terms of the Creative Commons Attribution License, which permits unrestricted use, distribution, and reproduction in any medium, provided the original author and source are credited. 